\title{
A pilot study of a cardiovascular virtual endoscopy system based on multi-detector computed tomography in diagnosing tetralogy of Fallot in pediatric patients
}

\author{
LI-PING YAO ${ }^{1,2}$, LI ZHANG ${ }^{2}$, JU MEI $^{2}$, FANG-BAO DING ${ }^{2}$, HUI-MING LI ${ }^{3}$, \\ MING DING ${ }^{3}$, XIN YANG ${ }^{4}, \mathrm{XIAO}-\mathrm{MING} \mathrm{LI}^{5}$ and KUN SUN ${ }^{1,6}$
}

\begin{abstract}
${ }^{1}$ Department of Biomedical Engineering, School of Biomedical Engineering, Shanghai Jiao Tong University, Shanghai 200030; Departments of ${ }^{2}$ Cardiothoracic Surgery and ${ }^{3}$ Radiology, Xinhua Hospital Affiliated to Shanghai Jiaotong University School of Medicine, Shanghai 200092; ${ }^{4}$ Department of Automation, Institute of Image Processing and Pattern Recognition, Shanghai Jiao Tong University, Shanghai 200240; ${ }^{5}$ Healthcare Department, Philips Research China, Shanghai 200233; ${ }^{6}$ Department of Pediatric Cardiology, Xinhua Hospital Affiliated to

Shanghai Jiaotong University School of Medicine, Shanghai 200092, P.R. China
\end{abstract}

Received July 5, 2016; Accepted April 28, 2017

DOI: $10.3892 /$ etm.2017.5572

\begin{abstract}
The present study aimed to investigate the capabilities of the cardiovascular virtual endoscopy (VE) system in diagnosing tetralogy of Fallot (TOF) and performing measurements. A total of 37 patients underwent two-dimensional echocardiography (2-DE) and multi-detector computed tomography (MDCT) examinations. The obtained MDCT images were applied to a cardiovascular VE system. Diagnostic time by VE was first studied and compared with MDCT. Subsequently, with surgical findings as the ground truth, the capabilities of VE, 2-DE and MDCT in diagnosing TOF and its complications were investigated. Additionally, measurements on aorta overriding ratio and diameters for the left pulmonary artery, right pulmonary artery and right ventricular outflow tract by 2-DE and VE were analyzed. Diagnostic time by VE was significantly shorter than MDCT $(188 \pm 42$ vs. $303 \pm 42 \mathrm{sec}$, respectively; $\mathrm{P}<0.0001)$. VE, MDCT and 2-DE demonstrated comparable diagnostic rates of TOF (35/37 vs. $34 / 37$ vs. $32 / 37$, respectively; $P>0.05$ ). Similar findings were demonstrated in diagnosing complications of the muscular ventricular septal defects, patent ductus arteriosus, vagus subclavian artery, right arch, double superior vena cava and pulmonary artery. Furthermore, in diagnosing the atrial septal defect, 2-DE outperformed MDCT and VE (accuracy, 100 vs. 81 vs. $73 \%$, respectively; all $\mathrm{P}<0.05$ ). In performing
\end{abstract}

Correspondence to: Professor Kun Sun, Department of Pediatric Cardiology, Xinhua Hospital Affiliated to Shanghai Jiaotong University School of Medicine, 1665 Kong-Jiang Road, Shanghai 200092, P.R. China

E-mail: sunkun@xinhuamed.com.cn

Key words: virtual endoscopy, roaming, cardiovascular, tetralogy of Fallot, 256-multislice computed tomography relevant measurements, VE outperformed MDCT and 2-DE, particularly in accessing aorta overriding ratios with no intra-operator difference $(\mathrm{P}=0.3770)$ and high consistency $(\mathrm{r}=0.916)$. In conclusion, cardiovascular VE was demonstrated to have acceptable accuracy in diagnosing TOF, and possess advantages in shortening the diagnostic time and in performing measurements.

\section{Introduction}

Tetralogy of Fallot (TOF) is the most common cyanotic congenital heart disease and is one of the most common causes of pediatric cyanosis $(1,2)$. TOF is usually a right-to-left shunt, in which higher resistance to right ventricular outflow results in more severe cyanosis symptoms (3). Furthermore, TOF is generally regarded to involve four heart anatomical abnormalities, which present together. These include right ventricular outflow tract obstruction (RVOTO) or pulmonary artery stenosis, ventricular septal defect (VSD), aortic root overriding and right ventricular hypertrophy; although other anomalies may have additional presentations (4).

Imaging modalities serve important roles in the diagnosis and preoperative evaluation of TOF. For years, echocardiography has been utilized as the first choice (5), with the advantages of real-time imaging, convenience and non-invasive (6). However, its performance is limited by factors, including obesity and due to it having a poor acoustic window (7). More recently, cardiac magnetic resonance has been established as an accurate imaging modality for cardiac anatomy and extra cardiac vascular structures; however, the examination is time consuming and difficult to perform in pediatric patients (7). With the development of computed tomography (CT) technology, cardiac CT (CCT) has been more widely applied in pediatric patients due to its high temporal and spatial resolution, high scanning speed and low radiation dose (8-10). However, the evaluation and diagnosis of TOF by current imaging modalities are all based on standard 
two-dimensional (2D) images, which highly depend on the experience of the operator.

Virtual endoscopy (VE) is an application of visual reality technology in medicine, in which cross-sectional medical images acquired by CT or magnetic resonance imaging (MRI) are utilized to reconstruct a three-dimensional (3D) environment similar to that seen through an endoscope $(11,12)$. To date, several VE systems have been developed and demonstrated to be important tools in diagnosis, as well as in preoperative planning and training (13-18). However, few studies have been conducted on the development of cardiovascular VE systems and its applications in pediatric patients.

The present study aimed to develop a cardiovascular VE system based on images from multi-detector CT (MDCT) and explore its utilities in the diagnosis and evaluation of TOF in pediatric patients. Its performance in diagnosis and in performing relevant measurements was further compared with those of two-dimensional echocardiography (2-DE) and MDCT.

\section{Materials and methods}

Study patients. The present retrospective study was approved by the Institutional Review Board of Xinhua Hospital (Shanghai, China). Written informed consent was obtained from all participants. Between December 2012 and March 2015 in Xinhua Hospital (Shanghai, China), 40 children with TOF, who were elected for surgical treatment and received 2-DE and MDCT cardiac examinations before surgeries were enrolled. In the present study, 37 were included and 3 cases were ruled out due to poor image quality. Patient characteristics are illustrated in Table I. The average age of participants was $35 \pm 39$ months (range, 1-146 months) and there were 23 were males and 14 females. The average volume CT dose index, dose-length product and effective radiation dose was $4.3 \mathrm{mGy}$ (range, 1.65-6.34 mGy), $58 \mathrm{mGy} \mathrm{cm} \mathrm{(range,} \mathrm{19.7-92.2} \mathrm{mGy} \cdot \mathrm{cm}$ ) and $1.4 \mathrm{mSv}$ (range, 0.84-1.92 mSv), respectively.

As confirmed by surgery, all 37 cases were accompanied by other complications, including two cases of muscular ventricular septal defect, 13 cases of atrial septal defect or patent foramen ovale, 17 cases of patent ductus arteriosus, 1 case of vagus subclavian artery, 7 cases of right arch, 4 cases of double superior vena cava and 1 case of pulmonary artery sling.

2-DE image acquisition and diagnosis. In the 2-DE ultrasound examination (Fig. 1), the parasternal short axis plane was acquired to detect RVOTO (Fig. 1A), while the parasternal long axis plane was acquired to evaluate aorta overriding, VSD and right ventricular hypertrophy. The vertical distance from the anterior wall of the aortic root to the top of the VSD, and the vertical distance from the top of the VSD to the posterior wall of the aortic root $\left(\mathrm{L}_{1}\right.$ and $\left.\mathrm{L}_{2}\right)$ were measured, as illustrated in Fig. 1D, to calculate the aorta overriding ratio. The suprasternal plane was also acquired to measure the diameters for the left pulmonary artery (LPA) and right pulmonary artery (RPA) at the first branches.

All ultrasound examinations (B-mode, M-mode and color Doppler), measurements and diagnoses were performed by two experienced ultrasound doctors, each with $>7$ years of experience in the diagnosis of cardiac diseases, at the Cardiac Ultrasound room of the Cardiothoracic Surgery Department at Xinhua Hospital Affiliated to Shanghai Jiaotong University School of Medicine (Shanghai, China). These doctors independently used the same iE33 Ultrasound System (Philips Medical Systems, Inc., Bothell, WA, USA) equipped with a S5-1 transducer (1.7-3.4 M) and a S3-8 (2.8-5.6 M). Generally, the S3-8 was used on younger children and the S5-1 was used for older children, for the limited penetration depth of S3-8. A diagnostic result was given when the findings of the two doctors were consistent. If any disagreement occurred, misdiagnosis would be considered. For measurements on the aorta overriding ratio, LPA and RPA, three measurements were performed for each patient and the average value was utilized for further analysis.

256-MDCT image acquisition and diagnosis. All MDCT scans of the heart were performed using a 256-MDCT scanner (Royal Philips, Amsterdam, The Netherlands) by the same radiologist, who has 10 years of experience in cardiac applications, under the step-and-shoot mode. The tube current and tube voltage were adjusted based on the body weight configuration strategy, in order to minimize the radiation dose. Other settings were the same for all scans, with a collimation of $0.625 \mathrm{~mm}$, rotation time of $270 \mathrm{msec}$, slice thickness of $0.9 \mathrm{~mm}$, reconstruction interval of $0.45 \mathrm{~mm}$ and tube voltage of $80 \mathrm{kV}$. The contrast agent Iohexol-300 (General Electronics, Atlanta, GA, USA) was injected using a Mallinckrodt binocular high-pressure syringe (Liebel-Flarsheim Company, Cincinnati, $\mathrm{OH}, \mathrm{USA}$ ) through the median cubital vein (injection dose, $2 \mathrm{ml} / \mathrm{kg}$; injection rate, $1.0-2.0 \mathrm{ml} / \mathrm{sec}$ for infants and $3.5-5 \mathrm{ml} / \mathrm{sec}$ for children). A region of interest (ROI) was selected on the descending aorta for bolus tracking in order to trigger the scanning. The automatic scanning was triggered in $4 \mathrm{sec}$ once the attenuation within that ROI was $>80$ Hounsfield units. The temporal window for data acquisition was set at $40-45 \%$ of the R-R interval, which is the interval between two adjacent $R$ waves in an electrocardiogram.

Cardiac View software (Extended Brilliance ${ }^{\mathrm{TM}}$ Workspace: V4.5.5.51035; Royal Philips) was applied on the CT images for post-processing, including curved planar reconstruction, volume rendering and maximum intensity projection (MIP). The diagnosis of TOF by CT was based on the Van Praagh approach (19), where the oblique sagittal image parallel to the main pulmonary artery was used to detect stenosis and access the shortest dimension of the right ventricular outflow tract (RVOT; Fig. 1B). The oblique sagittal MIP image was used to diagnose aortic overriding and calculate the aortic overriding ratio by measuring the vertical distance from the anterior wall of the aortic root to the top of the VSD, and the vertical distance from the top of the VSD to the posterior wall of the aortic root $\left(\mathrm{L}_{1}\right.$ and $\left.\mathrm{L}_{2}\right)$, as illustrated in Fig. 1E. The coronal MIP image parallel to the main pulmonary artery was employed to evaluate the pulmonary artery and perform measurements for LPA and RPA.

Two cardiovascular radiologists, each with $>10$ years of experience in diagnosing TOF, independently performed the diagnosis and measurements. A diagnostic result was given when the findings of the two doctors were consistent. If any disagreement occurred, misdiagnosis would be considered. 
Table I. Characteristics of the study subjects with tetralogy of Fallot.

\begin{tabular}{lc}
\hline Variable & P-value \\
\hline General information & \\
Total, $\mathrm{n}$ & 37 \\
Age, months $\mathrm{a}^{\mathrm{a}}$ & $35 \pm 39$ \\
Male, $\mathrm{n}(\%)$ & $23(62)$ \\
Radiation dose & \\
DLP, mGy $\mathrm{cm}^{\mathrm{a}}$ & $58 \pm 23$ \\
CTDIvol, mGy & \\
ED, mSva & $4.3 \pm 1.8$ \\
Complications, $\mathrm{n}(\%)$ & $1.4 \pm 0.7$ \\
Muscle of ventricular septal defect & \\
Atrial septal defect & $2(5)$ \\
Patent ductus arteriosus & $13(35)$ \\
Vagus subclavian artery & $17(46)$ \\
Right arch & $1(3)$ \\
Double superior vena cava & $7(19)$ \\
Pulmonary artery sling & $4(11)$ \\
\hline
\end{tabular}

${ }^{\text {a }}$ Data are presented as the mean \pm standard deviation. DLP, dose-length product; CTDIvol, computed tomography dose index; ED, effective dose.

For measurements on the aorta overriding ratio, LPA, RAP and RVOT, three measurements were performed for each patient and the average value was utilized for further analysis. The time for diagnosis was recorded for each radiologist.

Cardiovascular VE system. The cardiovascular VE system was developed as outlined in Fig. 2 (20,21). The acquired 3D CT image sequences were utilized as the system input. Subsequently, 3D reconstruction of the vessels and heart was performed. The roaming path in the gross vessels, left heart and right heart were generated automatically or semi-automatically with the user manually placing 2-5 seeds. Following this, the system reconstructed the surrounding field from the viewpoint on the roaming path, and displayed this in real-time.

When used for the diagnosis of TOF, the roaming path of right pulmonary artery to the right heart allowed the viewer to look straight into the RVOTO; and the VE system was able to automatically detect the stenosis and provided the shortest diameter of the RVOTO, as demonstrated in Fig. 1C. When the viewpoint was placed under the aortic valve, the left and right ventricles were able to be observed at the same time, and the VSD could be detected. From a view perpendicular to the ventricular septal, the longest diameters of the right and left ventricle could be measured, as demonstrated in Fig. 1F, and the aorta overriding ratio could be calculated. On the roaming path of the pulmonary branches, the shortest diameters for LPA and RPA could be calculated automatically.

Two pediatric cardiologists, each with $>10$ years of experience in cardiac applications, were trained to use the VE system, and performed the diagnosis and measurements.
Table II. Comparison of diagnostic time for tetralogy of Fallot by MDCT and VE.

\begin{tabular}{|c|c|c|c|}
\hline Variable & $\begin{array}{l}\text { Diagnostic } \\
\text { time, sec }{ }^{\mathrm{a}}\end{array}$ & $\begin{array}{l}\text { Diagnostic time } \\
\text { range, sec }\end{array}$ & P-value \\
\hline Observers of VE & & & 0.8968 \\
\hline Operator 1 & $188 \pm 43$ & $105-285$ & \\
\hline Operator 2 & $187 \pm 41$ & $105-276$ & \\
\hline VE vs. MDCT & & & $<0.0001$ \\
\hline VE & $188 \pm 42$ & $105-272$ & \\
\hline MDCT & $303 \pm 42$ & $247-390$ & \\
\hline
\end{tabular}

${ }^{\text {aData }}$ are presented as the mean \pm standard deviation. MDCT, multi-detector computed tomography; VE, virtual endoscopy.

A diagnostic result was given when the findings of the two doctors were consistent. When any disagreement occurred, misdiagnosis was considered. For measurements for the aorta overriding ratio, LPA, RAP and RVOT, three measurements were performed for each patient and the average value was utilized for further analysis. The time for diagnosis was recorded for each cardiologist.

Statistical analysis. Paired two-tailed t-tests were applied to determine the significance of the difference in diagnostic time between the different operators and between MDCT and VE. Furthermore, the difference in measurements of the aorta overriding ratio, LPA, RPA and RVOT between different operators and between different diagnostic methods were also determined. Chi-square tests were adopted to compare the detection rate for diseases amongst different diagnostic methods. $\mathrm{P}<0.05$ was considered to indicate a statistically significant difference. With surgical results as the ground truth, truth positive (TP), false positive (FP), truth negative (TN), false negative (FN) and accuracy were calculated to evaluate diagnostic performance amongst the different diagnostic methods. Furthermore, Pearson's correlation coefficient, $r$ was used to evaluate the correlation of measurements between the two operators. A strong correlation was considered if $0.700 \leq|r|<1.000$. A weak correlation was considered if $0.400 \leq|r|<0.700$. The box-and-whisker plot was employed to display the mean data distribution for measurements using different imaging modalities. MedCalc (10.4.7.0) software (MedCalc Software byba, Ostend, Belgium) was utilized to perform the analyses.

\section{Results}

Diagnostic time between the two operators by VE. As illustrated in Table II, the average diagnostic time for the two operators who used VE was 188 \pm 43 (range, 105-272 sec) and $187 \pm 41 \mathrm{sec}$ (range, 105-276 sec), respectively. No significant difference in diagnostic time was demonstrated between the two operators $(\mathrm{P}=0.8968)$.

As demonstrated in Table II, the average diagnostic time by VE and MDCT was 188 $\pm 42 \mathrm{sec}$ (range, 105-272 sec) and $303 \pm 42 \mathrm{sec}$ (range, 247-390 sec), respectively. A significantly 
Table III. Diagnostic rate of TOF by 2-DE, MDCT and VE.

Pathology type

\begin{tabular}{lccccc}
\cline { 3 - 5 } Modalities & TOF & Pulmonary artery stenosis & Aorta overriding & Ventricular septal defect & Hypertrophy \\
\hline 2-DE, $\mathrm{n}(\%)$ & $32(86)$ & $34(92)$ & $34(92)$ & $37(100)$ & $37(100)$ \\
MDCT, $\mathrm{n}(\%)$ & $34(92)$ & $36(97)$ & $34(92)$ & $37(100)$ & $37(100)$ \\
VE, $\mathrm{n}(\%)$ & $35(95)$ & $35(95)$ & $37(100)$ & $37(100)$ & $37(100)$
\end{tabular}

TOF, tetralogy of Fallot; 2-DE, two-dimensional echocardiography; MDCT, multi-detector computed tomography; VE, virtual endoscopy.
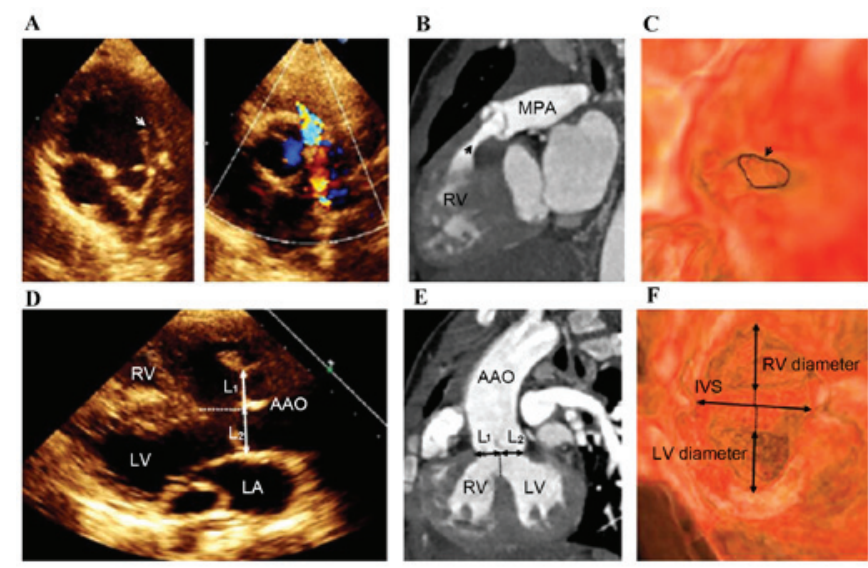

Figure 1. Diagnosis of TOF by 2-DE, MDCT and VE in a 2-year old female patient. The patient received corrective surgery. (A) Parasternal short-axis scan of 2-DE with (right panel) and without (left panel) color Doppler demonstrated stenosis of RVOT (white arrow). (B) Thick-section oblique sagittal MIP image of MDCT demonstrated RV hypertrophy and stenosis of RVOT (black arrow). (C) Direct sub-pulmonary view point of VE demonstrated stenosis of RVOT (black circle and arrow). (D) Parasternal long-axis scan of 2-DE demonstrated the VSD, overriding aorta and calculation for the overriding ratio $\left(\mathrm{L}_{2} / \mathrm{L}_{1}\right)$. (E) Thick-section oblique sagittal MIP image of MDCT demonstrated the VSD overriding aorta and the calculation for the overriding ratio $\left(\mathrm{L}_{2} / \mathrm{L}_{1}\right)$. (F) Direct sub-aorta view point by VE demonstrated two ventricles and the calculation for the overriding ratio (the longest LV diameter/RV diameter). TOF, tetralogy of Fallot; 2-DE, two-dimensional echocardiography; MDCT, multi-detector computed tomography; VE, virtual endoscopy; VSD, ventricular septal defect; RVOT, right ventricular outflow tract; MIP, maximum intensity projection; LV, left ventricular; RV, right ventricular; AAO, ascending aorta; MPA, main pulmonary artery; IVS, interventricular septum.

shorter diagnostic time was demonstrated when using VE as compared to that when using MDCT $(\mathrm{P}<0.0001)$.

Diagnostic rate of TOF amongst 2-DE, MDCT and VE. With surgical results as the ground truth, the overall diagnostic rate of TOF by 2-DE, MDCT and VE was 86 (32/37), 92 (34/37) and $95 \%$ (35/37), respectively. For the diagnosis of pulmonary artery stenosis, the diagnostic rate for 2-DE, MDCT and VE was 92 (34/37), 97 (36/37) and 95\% (35/37), respectively. For the diagnosis of aorta overriding, the diagnostic rate for 2-DE, MDCT and VE was 92 (34/37), 92 (34/37) and 100\% (37/37), respectively. For the diagnosis of ventricular septal defect and hypertrophy, the diagnostic rate for 2-DE, MDCT and VE were all 100\% (37/37), as demonstrated in Table III. Comparable diagnostic rate was observed amongst the three methods (all $\mathrm{P}>0.05$ ).

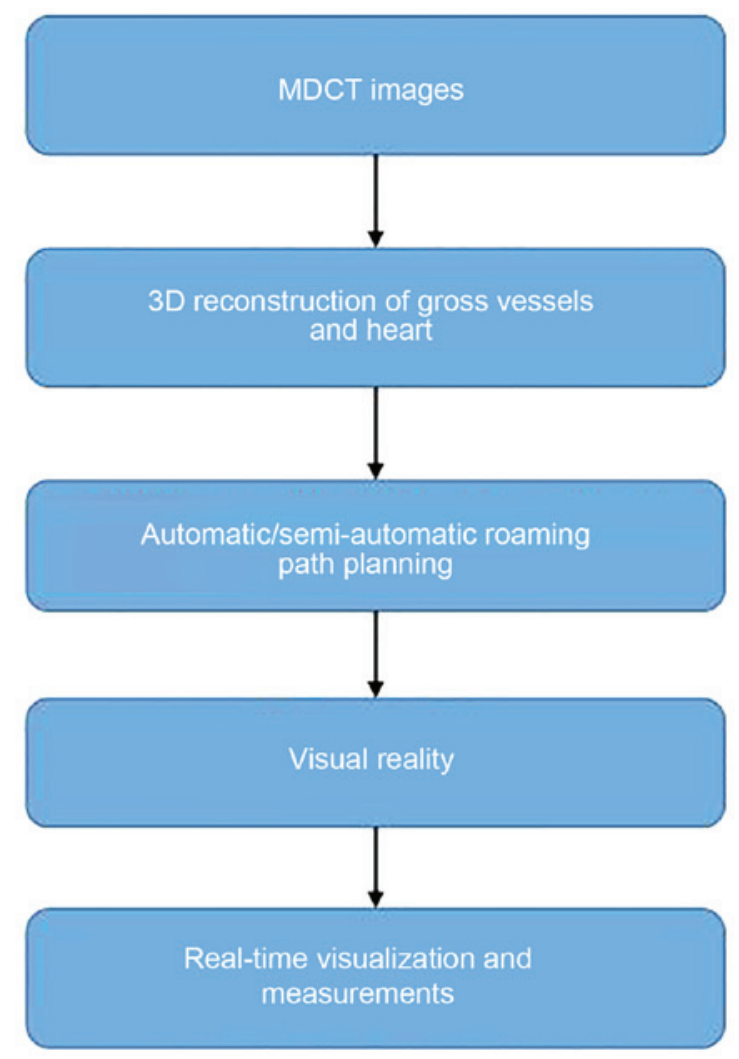

Figure 2. Framework for the cardiovascular virtual endoscopy system developed previously by the present research team $(20,21)$. MDCT, multi-detector computed tomography; 3D, three-dimensional.

Diagnostic rate of complications amongst 2-DE, MDCT and $V E$. The diagnostic performance for complications by 2-DE, MDCT and VE are illustrated in Table IV. For the diagnosis of muscular ventricular septal defect, 1 case was missed by MDCT (FN, 1/2; FP, 0/35) and VE (FN: 1/2; FP: 0/35), while this was correctly diagnosed by $2-\mathrm{DE}(\mathrm{FN}, 0 / 2 ; \mathrm{FP}, 0 / 35)$. Significantly higher accuracy was demonstrated when using 2-DE, as compared to MDCT and VE, for the diagnosis of the atrial septal defect $(\mathrm{P}<0.05)$, which had a diagnostic accuracy of 100 (FN, 0/13; FP, 0/24), 81 (FN, 6/13; FP, 1/24) and 73\% (FN, 8/13; FP, 2/24), respectively. However, for the diagnosis of patent ductus arteriosus, slightly higher accuracy was demonstrated by VE and MDCT, as compared with 2-DE, with an accuracy of 100 (FN, 0/17; FP, 0/20), 100 (FN, 0/17; FP, 0/20) and $95 \%$ (FN, 2/17; FP, 0/20), respectively. Similarly, the diagnostic accuracy of vagus subclavian artery by VE and MDCT 

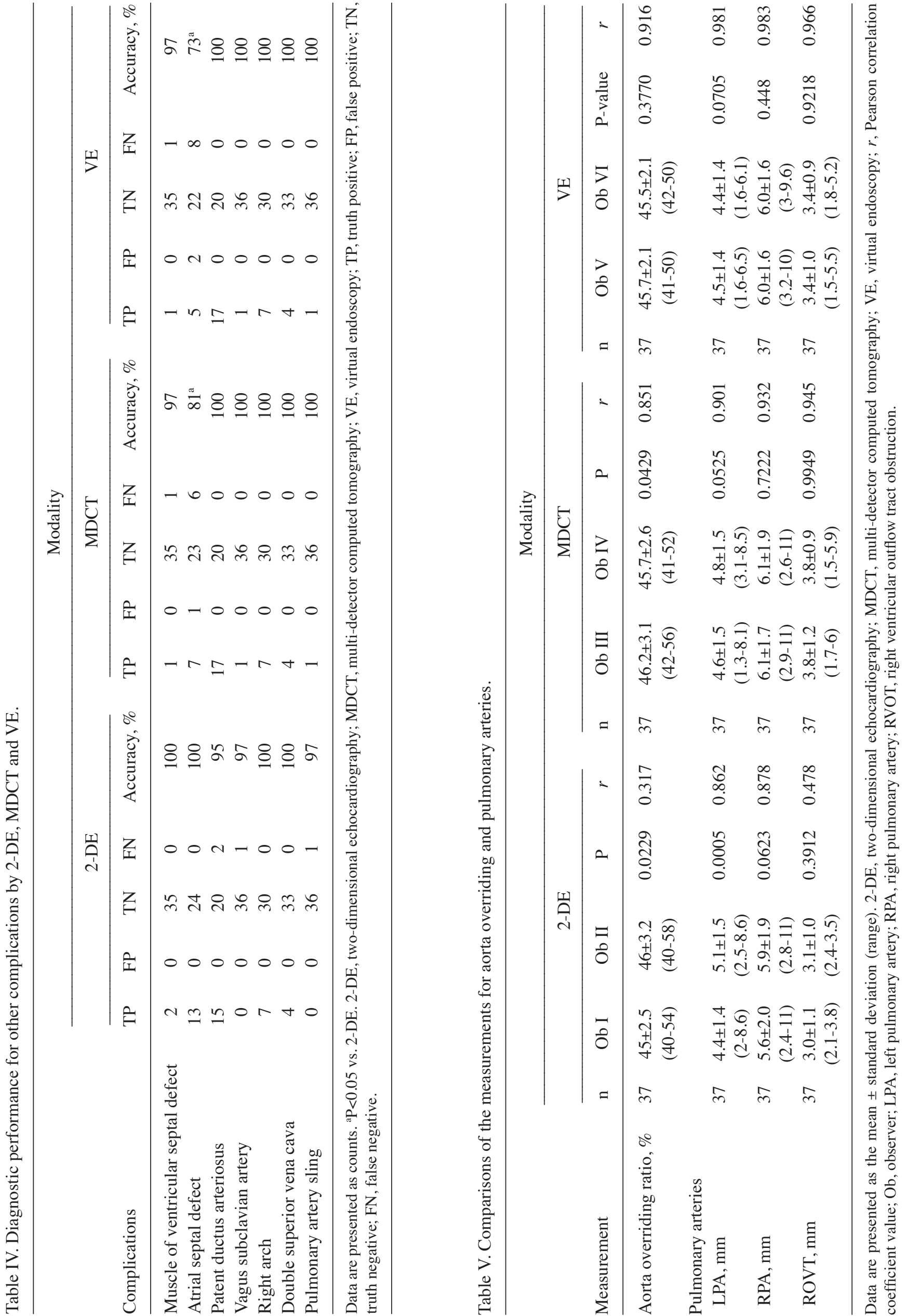
A

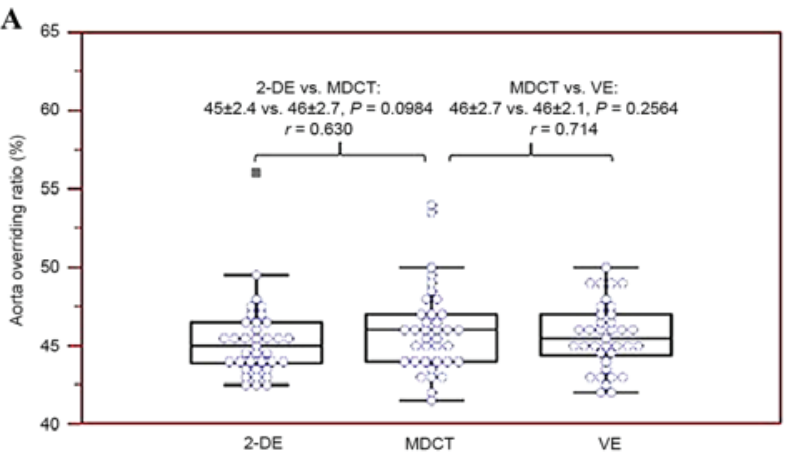

C

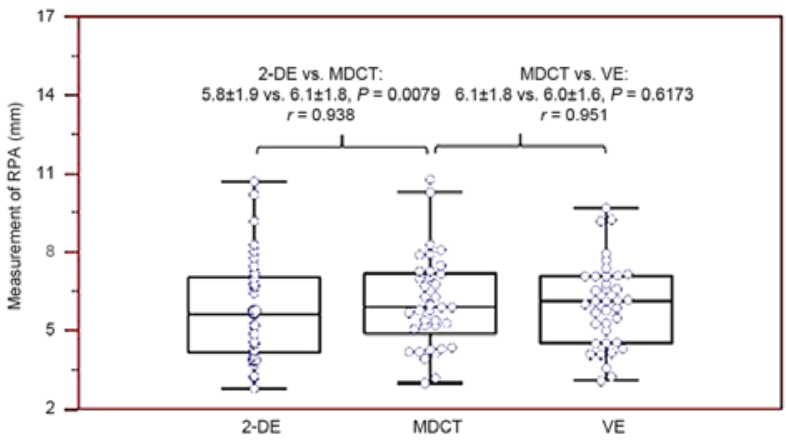

B

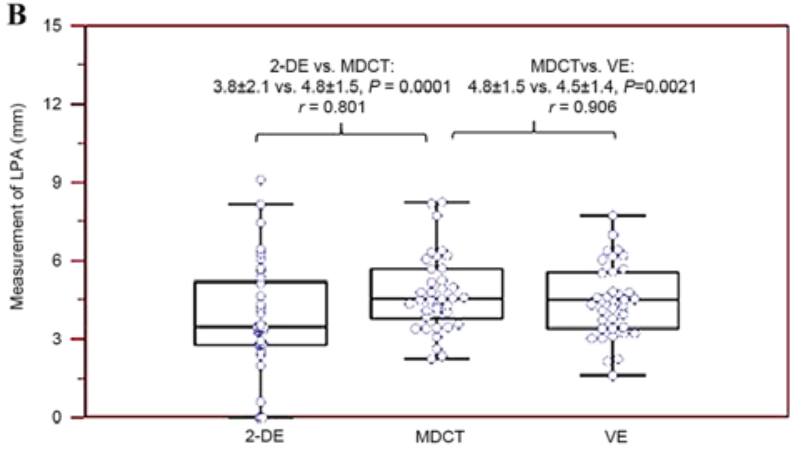

D

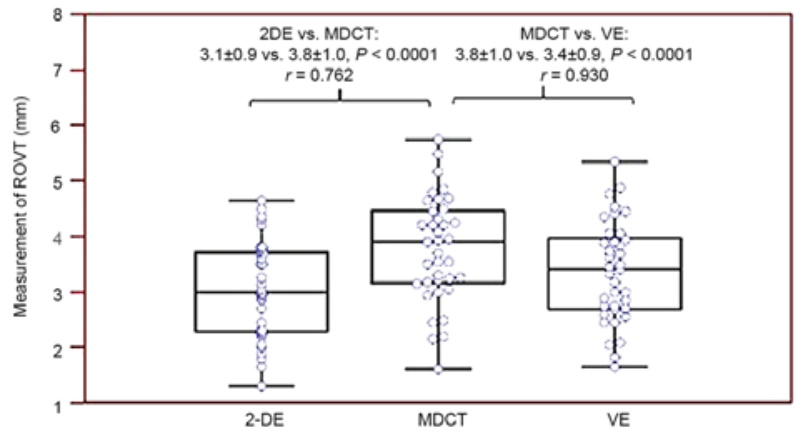

Figure 3. Measurements of aorta overriding ratio, LPA, RPA and RVOT by 2-DE, MDCT and VE. (A) Comparison of aorta overriding ratio by 2-DE, MDCT and VE. Measurements by VE had a slightly stronger correlation with those of MDCT as compared to 2-DE. (B) Comparison of LPA by 2-DE, MDCT and VE. Measurements by VE had a slightly stronger correlation with those of MDCT as compared to 2-DE. (C) Comparison of RPA by 2-DE, MDCT and VE. Measurements by VE and 2-DE had a comparable correlation with those of MDCT. (D) Comparison of RVOT by 2-DE, MDCT and VE. Measurements by VE had stronger correlation with those of MDCT as compared to 2-DE. LPA, left pulmonary artery; RPA, right pulmonary artery; RVOT, right ventricular outflow tract obstruction; 2-DE, two-dimensional echocardiography; MDCT, multi-detector computed tomography; VE, virtual endoscopy.

was slightly higher as compared with 2-DE, with an accuracy of $100(\mathrm{FN}, 0 / 1 ; \mathrm{FP}, 0 / 36), 100(\mathrm{FN}, 0 / 1 ; \mathrm{FP}, 0 / 36)$ and $97 \%$ (FN, 1/1; FP, 0/36), respectively. The diagnostic accuracy of the pulmonary artery sling was also slightly higher by VE and MDCT as compared to 2-DE, with an accuracy of 100 (FN, 0/1; FP, 0/36), 100 (FN, 0/1; FP, 0/36) and 97\% (FN, 1/1; FP, 0/36), respectively. Furthermore, for the right arch and double superior vena cava, a diagnostic accuracy of $100 \%$ (FN, 0/4; FP, 0/33) was observed for VE, MDCT and 2-DE.

Intra-operator study of measurements by 2-DE, MDCT and VE. The measurements of aorta overriding ratio, LPA, RPA and RVOT obtained by different operators using 2-DE, MDCT and VE are illustrated in Table V. For the evaluation of aorta overriding, significantly different overriding ratios were calculated between different operators by using either 2 -DE $(45 \pm 2.5$ vs. $46 \pm 3.2 ; \mathrm{P}=0.0229)$ or MDCT (46.2 \pm 3.1 vs. 45.7 $\pm 2.6 ; \mathrm{P}=0.0429)$. Furthermore, comparable overriding ratios were demonstrated between two different operators by using VE (45.7 \pm 2.1 vs. 45.5 \pm 2.1$)$. A strong correlation was observed between the measurements of overriding ratios between the different operators for both MDCT $(r=0.851)$ and VE $(r=0.916)$, while no correlation $(r=0.317)$ was observed between the two different operators when using 2-DE.

For the assessment of LPA, comparable measurements were demonstrated between different operators using MDCT $(4.6 \pm 1.5$ vs. $4.8 \pm 1.5 \mathrm{~mm} ; \mathrm{P}=0.0525)$ and VE $(4.5 \pm 1.4$ vs. $4.4 \pm 1.4 \mathrm{~mm} ; \mathrm{P}=0.0705)$, while significantly different measurements were observed between different operators using 2 -DE ( $4.4 \pm 1.4$ vs. $5.1 \pm 1.5 \mathrm{~mm} ; \mathrm{P}=0.0005)$. Furthermore, a strong correlation was demonstrated between the measurements of LPA between the different operators for 2-DE, MDCT and VE, respectively, with all $r>0.7$.

For the assessment of RPA, comparable measurements were observed between the different operators when 2-DE $(5.6 \pm 2.0$ vs. $5.9 \pm 1.9 \mathrm{~mm} ; \mathrm{P}=0.0623)$, MDCT $(6.1 \pm 1.7$ vs. $6.1 \pm 1.9 \mathrm{~mm} ; \mathrm{P}=0.7222)$ and $\mathrm{VE}(6.0 \pm 1.6 \mathrm{vs} .6 .0 \pm 1.6 \mathrm{~mm}$; $\mathrm{P}=0.448$ ) was used. Furthermore, a strong correlation was demonstrated between the measurements of RPA between the different operators for 2-DE, MDCT and VE, respectively, with all $r>0.7$.

For the assessment of RVOT, comparable measurements were observed between the different operators when 2-DE $(3.0 \pm 1.1$ vs. $3.1 \pm 1.0 \mathrm{~mm} ; \mathrm{P}=0.3912)$, MDCT (3.8 \pm 1.2 vs. $3.8 \pm 0.9 \mathrm{~mm} ; \mathrm{P}=0.9949)$ and $\mathrm{VE}(3.4 \pm 1.0$ vs. $3.4 \pm 0.9 \mathrm{~mm}$; $\mathrm{P}=0.9218)$ were used. Furthermore, a strong correlation was demonstrated between the measurements of LPA for different operators for both MDCT and VE, with all $r>0.7$. However, a weak correlation was observed between the two different operators when 2-DE ( $r=0.478$;) was used.

Comparison of measurements by 2-DE and VE to MDCT. The comparison in measurements of aorta overriding ratio, LPA, RPA and RVOT by different methods is illustrated in Fig. 3. As compared to MDCT, no significant difference in measurements for the aorta overriding ratio were demonstrated by 
either 2-DE $(45 \pm 2.4$ vs. $46 \pm 2.7 ; \mathrm{P}=0.0984)$ or VE $(46 \pm 2.7$ vs. $46 \pm 2.1 ; \mathrm{P}=0.2564)$. However, measurements for the aorta overriding ratio by $\mathrm{VE}$ had a slightly stronger correlation with that obtained by MDCT, as compared with 2-DE ( $r, 0.714$ vs. $0.630)$.

For measurements of LPA, and RVOT, significantly different measurements were demonstrated between 2-DE or VE, compared with MDCT (all P>0.05). The differences in RPA measurement between 2-DE and MDCT were significant $(\mathrm{P}=0.0079)$, while no significant difference was indicated between VE and MDCT. VE demonstrated a slightly stronger correlation on the measurements of LPA and RPA with MDCT, as compared with 2-DE. For the measurements of RVOT, VE had a significantly stronger correlation with MDCT, as compared to $2 \mathrm{DE}$ ( $r, 0.930$ vs. 0.762$)$.

\section{Discussion}

Effective imaging technologies provide accurate, reliable and convenient tools for the diagnosis of diseases, pre-operational evaluation and surgical planning. VE is a visual reality technology that is usually based on X-ray, CT and MRI images. Its utility has been investigated in cardiac applications in adults $(15,22-24)$. However, very few studies have explored its utilities in pediatric applications. Most recently, with the development of CT technology, high temporal and special resolution images for pediatric hearts and gross vessels could be acquired (8-10). Therefore, in the present study, based on MDCT technology, a cardiovascular VE system was developed and applied to the diagnosis and evaluation of TOF in 37 pediatric patients. Its reproducibility and performance in diagnosis and measurements were then compared with those of in 2-DE and MDCT.

In the present study, consistent diagnostic results were obtained by two different operators who used VE, and no difference in diagnostic time was observed. The mean diagnostic time between VE and MDCT was also compared, and VE demonstrated a significantly shorter diagnostic time. Regarding the overall diagnostic rate of TOF, a comparable diagnostic rate was observed when using $\mathrm{VE}$, as compared to MDCT and 2-DE. For the diagnosis of ventricular septal defect and hypertrophy, all patients were correctly diagnosed by VE, MDCT and 2-DE. For the diagnosis of aorta overriding, the performance of VE was slightly better than that of MDCT and 2-DE. For the diagnosis of pulmonary artery stenosis, 1 case was missed by VE, while this was correctly diagnosed by MDCT. We further reviewed this case and found that branches of the pulmonary arteries of this patient were very narrow, and that the VE system ignored these vessels while performing the automatic reconstruction. In this situation, the semi-automatic method should be used.

In terms of the diagnosis of other complications of muscular ventricular septal defect, patent ductus arteriosus, vagus subclavian artery, right arch, double superior vena cava and pulmonary artery sling, a comparable diagnostic accuracy was demonstrated for VE, MDCT and 2-DE. However, a significantly higher diagnostic accuracy of atrial septal defect was demonstrated by 2-DE, as compared to MDCT and VE. This was induced by the intrinsic limitation of cardiac CT technology, in which artifacts appear for the imaging of thinner and moving targets. The present VE system was developed based on MDCT technology. Therefore, its performance on the diagnosis of atrial septal defects was also influenced.

Relevant measurements, including aortic overriding ratio, as well as the diameters of LPA, RPA and RVOT, are important in the diagnosis and treatment for TOF patients, particularly for the assessment of the aorta overriding ratio. TOF would be diagnosed with an aorta overriding ratio of $\leq 50 \%$, while double outlet of right ventricle would be diagnosed for an aorta overriding ratio of $>50 \%$ (25). Different interventions were applied. In the present study, VE performed best in accessing the aorta overriding ratio with no intra-observer difference and higher measurement consistency, while significantly different aorta overriding ratios were obtained by different operators by MDCT and 2-DE. This was due to the fact that 2D scans were utilized to perform measurements by either MDCT or 2-DE. The acquisition of these 2-DE planes were operator dependent; therefore, influencing accuracy and measurement consistency. For the VE system, 3D information was utilized. Regarding the diameters of LPA, RPA and RVOT, VE slightly out-performed MDCT with higher measurement consistency. Furthermore, 2-DE had the worst performance due to its limited acoustic window and consequent image with poor quality when accessing pulmonary arteries.

The present study was unique in several aspects. First, a cardiovascular VE system was developed based on MDCT images obtained from pediatric patients with TOF, and its clinical utilities in diagnosing TOF and its compilations were investigated and compared with MDCT and 2-DE. Second, the performance of VE, MDCT and 2-DE in accessing aorta overriding ratios and the diameters of LPA, RPA and RVOT were further studied and compared. The present study also had disadvantages. A total of only 37 cases were involved. More patients and more complications should be included. Furthermore, there was a lack of ground truth measurements from surgeries, as these are not routinely performed in clinical practice.

In conclusion, cardiovascular VE had a comparable performance to MDCT and 2-DE in TOF diagnosis. It was performed in shorter diagnostic time than MDCT when diagnosing TOF and complications. VE performed better in accessing related measurements, as compared to MDCT and 2-DE, with higher measurement consistency between different operators. Therefore, VE may be a valuable tool in the diagnosis and evaluation of TOF.

\section{Acknowledgements}

The present study was supported by the Key Project for the Shanghai Committee of Science and Technology Basic Research (grant no. 12JC1406600) and the Research Project of Shanghai Municipal Commission of Health and Family Planning (grant no. 201540135).

\section{References}

1. Breitbart R and Fyler D: Nadas' Pediatric Cardiology. Saunders-Elsevier, Philadelphia, PA, pp559, 2006.

2. Keane JF, Lock JE and Fyler DC: Tetralogy of fallot. In: Nadas' Pediatric Cardiology. 2nd edition. Saunders-Elsevier, Philadelphia, PA, pp559, 2006. 
3. Kumar V, Abbas A and Aster J: Robbins Basic Pathology. 9th edition. Elsevier/Saunders, Philadelphia, PA, pp365-406, 2013.

4. Anderson RH, Spicer DE, Giroud JM and Mohun TJ: Tetralogy of Fallot: Nosological, morphological, and morphogenetic considerations. Cardiol Young 23: 858-866, 2013.

5. Bogarapu S, Pinto NM, Etheridge SP, Sheng X, Liesemer KN, Young PC and Saarel EV: Screening for congenital heart disease in infants with down syndrome: Is universal echocardiography necessary? Pediatr Cardiol 37: 1222-1227, 2016.

6. White SK, Sado DM, Flett AS and Moon JC: Characterising the myocardial interstitial space: The clinical relevance of non-invasive imaging. Heart 98: 773-779, 2012

7. Gao Y, Lu B, Hou Z, Yu F, Cao H, Han L and Wu R: Low dose dual-source CT angiography in infants with complex congenital heart disease: A randomized study. Eur J Radiol 81: e789-e795, 2012.

8. Cui Y, Huang M, Zheng J, Li J, Liu H and Liang C: Assessments of coronary artery visibility and radiation dose in infants with congenital heart disease on cardiac 128-slice CT and on cardiac 64-slice CT. Pediatr Cardiol 37: 135-143, 2016.

9. Zhang T, Wang W, Luo Z, Wang D, Bai J, Han D and Shen B: Initial experience on the application of 320-row CT angiography with low-dose prospective ECG-triggered in children with congenital heart disease. Int J Cardiovasc Imaging 28: 1787-1797, 2012.

10. Nie P, Wang X, Cheng Z, Ji X, Duan Y and Chen J: Accuracy, image quality and radiation dose comparison of high-pitch spiral and sequential acquisition on 128-slice dual-source CT angiography in children with congenital heart disease. Eur Radiol 22 : 2057-2066, 2012.

11. Wood BJ and Razavi P: Virtual endoscopy: A promising new technology. Am Fam Physician 66: 107-112, 2002.

12. Lim F, Brown I, McColl R, Seligman C and Alsaraira A: Conf Proc IEEE Eng Med Biol Soc I: 1513-1516, 20016.

13. Triantafyllou K, Lazaridis LD and Dimitriadis GD: Virtual reality simulators for gastrointestinal endoscopy training. World J Gastrointest Endosc 6: 6-12, 2014.

14. Di Somma A, de Notaris M, Stagno V, Serra L, Enseñat J, Alobid I, San Molina J, Berenguer J, Cappabianca P and Prats-Galino A: Extended endoscopic endonasal approaches for cerebral aneurysms: Anatomical, virtual reality and morphometric study. Biomed Res Int 2014: 703792, 2014.

15. Terada Y, Sekii H and Ichikawa S: Virtual intraaortic endoscopy in severe calcific aortic stenosis. Ann Thorac Surg 91: 1291, 2011.
16. Wong KY, Tse HN, Pak KK, Wong CC, Lok PS, Chan KC and Yee WK: Integrated use of virtual bronchoscopy and endobronchial ultrasonography on the diagnosis of peripheral lung lesions. J Bronchology Interv Pulmonol 21: 14-20, 2014.

17. Orabi H, Aboushwareb T, Tan J, Yoo JJ and Atala A: Can computed tomography-assisted virtual endoscopy be an innovative tool for detecting urethral tissue pathologies? Urology 83: 930-938, 2014

18. Alotaibi FE, AlZhrani GA, Mullah MA, Sabbagh AJ, Azarnoush H, Winkler-Schwartz A and Del Maestro RF: Assessing bimanual performance in brain tumor resection with NeuroTouch, a virtual reality simulator. Neurosurgery 11 (Suppl 2): S89-S98, 2015.

19. Watts JR Jr, Sonavane SK, Singh SP and Nath PH: Pictorial review of multi detector $\mathrm{CT}$ imaging of the preoperative evaluation of congenital heart disease. Curr Probl Diagn Radiol 42: 40-56, 2013.

20. Du A, Yang X, Xue H, Yao L and Sun K: 3D virtual endoscopy of heart. Sheng Wu Yi Xue Gong Cheng Xue Za Zhi 29: 974-982, 2012 (In Chinese).

21. Li J, Yang X, Yao LP and Sun K: 3-D centerline extraction for virtual cardiovascular endoscopy. Conference: 2014 11th International Computer Conference on Wavelet Active Media Technology and Information Processing (ICCWAMTIP). DOI: 10.1109/ICCWAMTIP.2014.7073380.

22. Nyamse V, Charissis V, Moore JD, Parker C, Khan S and Chan W: The design considerations of a virtual reality application for heart anatomy and pathology education virtual, augmented and mixed reality. Systems and Applications Volume 8022 of the series Lecture Notes in Computer Science, pp66-73, 2013.

23. Chen GC, Lin CH, Li CM, Hsieh KS, Du YC and Chen T: Virtual-Reality simulator system for double interventional cardiac catheterization using fractional-order vascular access tracker and haptic force producer. Scientific World J 2015: 12 , 2015.

24. Jensen K, Bjerrum F, Hansen HJ, Petersen RH, Pedersen JH and Konge L: A new possibility in thoracoscopic virtual reality simulation training: Development and testing of a novel virtual reality simulator for video-assisted thoracoscopic surgery lobectomy. Interact Cardiovasc Thorac Surg 21: 420-426, 2015.

25. Raju V, Burkhart HM, Rigelman Hedberg N, Eidem BW, Li Z, Connolly H, Schaff HV and Dearani JA: Strategy for atrioventricular septal defect and tetralogy of Fallot or double-outlet right ventricle. Ann Thorac Surg 95: 2079-2085, 2013. 